\title{
WIENER-HOPF OPERATORS ON A FINITE INTERVAL AND SCHATTEN-VON NEUMANN CLASSES
}

\author{
VLADIMIR V. PELLER
}

(Communicated by John B. Conway)

\begin{abstract}
Recently R. Rochberg has characterized Hankel and Toeplitz operators on the Paley-Wiener space which belong to the Schatten-von Neumann class $S_{p}$ for $p \geq 1$. These operators coincide up to Fourier transform with Wiener-Hopf operators on a finite interval. Using a different approach, we extend Rochberg's result to all positive $p$.
\end{abstract}

1. Introduction. In this paper we investigate the integral operators $W_{\sigma, k}$ on $L^{2}[-\sigma, \sigma]$ defined by

$$
\left(W_{\sigma, k} f\right)(x)=\int_{-\sigma}^{\sigma} k(x-y) f(y) d y, \quad x \in[-\sigma, \sigma],
$$

where $k$ is a function (or a distribution) on $\mathbb{R}$. These operators are analogues of Wiener-Hopf operators which are defined on $L^{2}\left(\mathbf{R}_{+}\right)$. They are important in different domains of analysis.

The main purpose of the paper is to obtain a necessary and sufficient condition on $k$ in order that $W_{\sigma, k}$ be in the Schatten-von Neumann class $\mathbb{S}_{p}, 0<p<\infty$.

Recall that $\boldsymbol{S}_{p}$ consists of the operators $T$ on a Hilbert space whose singular numbers $s_{n}(T)$ (i.e. the eigenvalues of $\left(T^{*} T\right)^{1 / 2}$ arranged in the nonincreasing order) satisfy the condition

$$
\|T\|_{s_{p}}=\left(\sum_{n \geq 0}\left(s_{n}(T)\right)^{p}\right)^{1 / p}<\infty .
$$

We refer the reader to $[\mathbf{1}]$ for information about $\boldsymbol{S}_{p}$-classes. We only mention here that if $A, B$ are bounded operators and $T \in \mathbb{S}_{p}$ then $A T B \in \mathbb{S}_{p}$ and

$$
\|A T B\|_{\mathrm{s}_{p}} \leq\|A\| \cdot\|T\|_{\mathrm{s}_{p}} \cdot\|B\|
$$

(here $\|\cdot\|$ is the operator norm).

Besides the operators $W_{\sigma, k}$ one can consider truncated continual Hankel operators $\Gamma_{\sigma, k}$ on $L^{2}[-\sigma, \sigma]$ defined by

$$
\left(\Gamma_{\sigma, k} f\right)(x)=\int_{-\sigma}^{\sigma} k(x+y) f(y) d y, \quad x \in[-\sigma, \sigma] .
$$

Received by the editors September 10, 1987 and, in revised form, October 27, 1987. The contents of this paper have been presented at the postconference of the U.S.-Sweden Seminar Function spaces and applications, Sund, Sweden, June 1986.

1980 Mathematics Subject Classification (1985 Revision). Primary 47B35, 47B10, 47G05; Secondary 46E35.

Key words and phrases. Wiener-Hopf operator, Hankel operator, Toeplitz operator, Schattenvon Neumann classes. 
Though the properties of Wiener-Hopf operators and Hankel operators are quite different, the operators $\Gamma_{\sigma, k}$ and $W_{\sigma, k}$ differ from each other by a unitary factor. Indeed, let $U$ be the unitary operator on $L^{2}[-\sigma, \sigma]$ defined by

$$
(U f)(x)=f(-x), \quad x \in[-\sigma, \sigma] .
$$

Then $\Gamma_{\sigma, k}=W_{\sigma, k} U$. So $\Gamma_{\sigma, k} \in \mathbb{S}_{p}$ if and only if $W_{\sigma, k} \in \mathbb{S}_{p}$.

As in the case of Wiener-Hopf operators and Hankel operators, the operators $W_{\sigma, k}$ (and $\Gamma_{\sigma, k}$ ) can be expressed in terms of Fourier transform as compressions of multiplication operators. Indeed, by the Paley-Wiener theorem (see [2]) the Fourier transform $\mathscr{F}$ of $\left\{f \in L^{2}(\mathbb{R}): \operatorname{supp} f \subset[-\sigma, \sigma]\right\}$ is the space $\mathrm{PW}_{\sigma}$ which consists of the entire functions of exponential type at most $\sigma$ whose restrictions to the real line belong to $L^{2}(\mathbb{R})$. Thus, the Paley-Wiener theorem reduces the operators $W_{\sigma, k}$ to the operators $T_{\sigma, \varphi}$ on $\mathrm{PW}_{\sigma}$ defined by $T_{\sigma, \varphi} f=\mathbb{P}_{\sigma} \varphi f$, where $\mathbb{P}_{\sigma}$ is the orthogonal projection from $L^{2}(\mathbb{R})$ onto $\mathrm{PW}_{\sigma}$.

In a recent paper [6] $\mathrm{R}$. Rochberg described the bounded operators $W_{\sigma, k}$ and the operators $W_{\sigma, k}$ of class $\mathbb{S}_{p}$ for $1 \leq p \leq \infty$ in terms of the symbol $k$. Note that for $0<p<1,\|\cdot\|_{S_{p}}$ defined by (1) is not a norm, $\mathbb{S}_{p}$ is not a Banach space, and Rochberg's techniques do not work in this case.

We suggest another approach here which permits us to cover all values of $p$.

Note that R. Rochberg in [6] also described the operators $W_{\sigma, k}$ of finite rank and found interesting relations between the operators $W_{\sigma, k}$ and the discrete commutator operators, i.e. the operators on $l^{2}(\mathbb{Z})$ with matrices

$$
a_{j, k}= \begin{cases}\left(c_{j}-c_{k}\right) /(j-k), & j \neq k, \\ 0, & j=k,\end{cases}
$$

where $\left\{c_{n}\right\}$ is a sequence of complex numbers.

We shall reduce the problem of description of operators $W_{\sigma, k}$ of class $\mathbb{S}_{p}$ to the corresponding problem for Hankel operators. Given a function (or distribution) $k$ on $\mathbb{R}_{+}$, the Hankel operator $\Gamma_{k}$ on $L^{2}\left(\mathbb{R}_{+}\right)$is defined by

$$
\left(\Gamma_{k} f\right)(x)=\int_{0}^{\infty} k(x+y) f(y) d y, \quad x \in \mathbb{R}_{+} .
$$

Now we recall the description of the Hankel operators of class $\mathbb{S}_{p}$.

THEOREM. Let $0<p<\infty$. The operator $\Gamma_{k}$ belongs to $\mathbb{S}_{p}$ if and only if the Fourier transform $\mathscr{F} k$ of its symbol belongs to the Besov space $B_{p}=B_{p}^{1 / p}(\mathbb{R})$.

See $[\mathbf{4}]$ for $1 \leq p<\infty$ and $[\mathbf{5}, \mathbf{8}]$ for $p<1$.

We refer the reader to [3] for basic properties of Besov spaces which admit many equivalent definitions. We only recall one of them.

Let $\left\{\psi_{j}\right\}_{j \in \mathbf{Z}}$ be $C^{\infty}$-functions on $\mathbf{R}_{+}$such that $\operatorname{supp} \psi_{j} \subset\left[2^{j-1}, 2^{j+1}\right], 0 \leq \psi_{j} \leq$ $1, \sum_{j \in \mathbf{Z}} \psi_{j}(x) \equiv 1$ on $\mathbb{R}_{+}$and $\psi_{j+1}(x)=\psi_{j}(x / 2)$. It is very easy to construct such a system of functions. Then $\mathscr{F} k \in B_{p}$ if and only if

$$
\sum_{j \in \mathbb{Z}} 2^{j}\left\|\mathscr{F}\left(\psi_{j} k\right)\right\|_{L^{p}(\mathbf{R})}^{p}<\infty .
$$

Now we are going to formulate the main result of the paper. 
Let $I=[\alpha, \beta]$ be an interval. We associate with $I$ a system of functions $\left\{\nu_{j}\right\}_{j \in \mathbb{Z}}$ in the following way:

$$
\begin{aligned}
& \nu_{j}(x)=\psi_{j}(2(x-\alpha) /(\beta-\alpha)), \quad j<0 \\
& \nu_{j}(x)=\nu_{j}(\alpha+\beta-x), \quad j>0 \\
& \nu_{0}(x)=1-\sum_{j \neq 0} \nu_{j}(x)
\end{aligned}
$$

Clearly, $0 \leq \nu_{j} \leq 1, \operatorname{supp} \nu_{j} \subset[\alpha,(\alpha+\beta) / 2]$ for $j<0$ and $\operatorname{supp} \nu_{j} \subset[(\alpha+\beta) / 2, \beta]$ for $j>0$.

THEOREM 1. Let $\left\{\nu_{j}\right\}_{j \in \mathbb{Z}}$ be the sequence of functions associated with $[-2 \sigma, 2 \sigma]$. Then $W_{\sigma, k} \in \mathbb{S}_{p}$ if and only if

$$
\sum_{j \in \mathbf{Z}} 2^{-|j|}\left\|\mathscr{F}\left(\nu_{j} k\right)\right\|_{L^{p}}^{p}<\infty .
$$

Note that this description resembles the description of operators $\Gamma_{k}$ of class $\mathbb{S}_{p}$ if we rewrite (2) as

$$
\sum_{j \in \mathbf{Z}}\left|\operatorname{supp} \psi_{j}\right| \cdot\left\|\mathscr{F}\left(\psi_{j} k\right)\right\|_{L^{p}}^{p}<\infty
$$

and rewrite (3) as

$$
\sum_{j \in \mathbb{Z}}\left|\operatorname{supp} \nu_{j}\right| \cdot\left\|\mathscr{F}\left(\nu_{j} k\right)\right\|_{L^{p}}^{p}<\infty
$$

Denote by $\mathrm{WH}_{p}$ the space of those symbols $k$ for which $W_{\sigma, k} \in \mathbb{S}_{p}$. In $\S 3$ answering a question by Rochberg [6], we show that the spaces $\mathrm{WH}_{p}$ form an interpolating scale.

2. Proof of the main result. In this section we establish the main result of the paper. It is more convenient for us to work with truncated Hankel operators on the interval $[0, a]$. So, given a function $k$, we consider the integral operator $\Gamma_{k}^{a}$ on $L^{2}[0, a]$ defined by

$$
\left(\Gamma_{k}^{a} f\right)(x)=\int_{0}^{a} k(x+y) f(y) d y, \quad x \in[0, a] .
$$

Obviously $\Gamma_{k}^{a}$ depends only on the restriction of $k$ to the interval $[0,2 a]$. Consider the sequence $\left\{\nu_{j}\right\}_{j \in \mathbf{Z}}$ associated with the interval $[0,2 a]$. It is easy to see that Theorem 1 stated in $\S 1$ is equivalent to the following assertion.

THEOREM 2. Let $0<p<\infty$. The operator $\Gamma_{k}^{a}$ belongs to the class $\mathbb{S}_{p}$ if and only if

$$
\sum_{j \in \mathbf{Z}} 2^{-|j|}\left\|\mathscr{F}\left(\nu_{j} k\right)\right\|_{L^{p}}^{p}<\infty .
$$

As we have mentioned in $\S 1$, for $1 \leq p<\infty$ this result has been obtained by Rochberg [6].

We shall reduce the investigation of the operators $\Gamma_{k}^{a}$ to the case of Hankel operators. If $\operatorname{supp} k \subset[0, a]$, we can do this straightforwardly. Indeed, in this 
case $\Gamma_{k}^{a}$ is exactly the restriction of the Hankel operator $\Gamma_{k}$ to $L^{2}[0, a]$. Moreover $\Gamma_{k} \mid L^{2}[a, \infty)=\mathbf{0}$. Therefore $\Gamma_{k}^{a} \in \mathbb{S}_{p}$ if and only if $\Gamma_{k} \in \mathbb{S}_{p}$ which is equivalent to

$$
\sum_{j \leq 0} 2^{-|j|}\left\|\mathscr{F}\left(\nu_{j} k\right)\right\|_{L^{p}}^{p}<\infty
$$

(see $\S 1$. The same can be done if $\operatorname{supp} k \subset[a, 2 a]$. Indeed, in this case $\Gamma_{k}^{a}$ is unitarily equivalent to the operator $\Gamma_{\tilde{k}}^{a}$ where $\tilde{k}(x) \stackrel{\text { def }}{=} k(2 a-x)$ (the unitary equivalence is given by the reflection operator $U$ defined by $\left.(U f)(x)=f(a-x), f \in L^{2}[0, a]\right)$. Clearly $\operatorname{supp} \tilde{k} \subset[0, a]$. So in this case $\Gamma_{k}^{a} \in \mathbb{S}_{p}$ if and only if

$$
\sum_{j \geq 0} 2^{-|j|}\left\|\mathscr{F}\left(\nu_{j} k\right)\right\|_{L^{p}}^{p}<\infty .
$$

Now, if $1<p<\infty$, the proof can easily be completed if we apply the theorem on the boundedness of the triangular projection on $\boldsymbol{S}_{p}, 1<p<\infty$ (see [1]). Indeed, if this projection is bounded, we can consider two functions $r=\chi_{[0, a]} k$ and $s=\chi_{[a, 2 a]} k$ and it follows that $\Gamma_{k}^{a} \in \mathbb{S}_{p}$ if and only if both $\Gamma_{r}^{a}$ and $\Gamma_{s}^{a}$ belong to $\mathbb{S}_{p}$. Now estimates (4) and (5), applied to $\Gamma_{r}^{a}$ and $\Gamma_{s}^{a}$ respectively, give the desired result.

In the proof of Theorem 2 we shall suppose that $0<p \leq 1$. Nevertheless it also works for $1<p<\infty$ and in the last case it is much simpler. So, actually we do not need the theorem on the triangular projection.

In the case $0<p \leq 1$ the above cutting $\Gamma_{k}^{a}=\Gamma_{r}^{a}+\Gamma_{s}^{a}$ does not work. In order to reduce Theorem 2 in this case to Hankel operators we shall cut the symbol $k$ in a more delicate way.

Consider $C^{\infty}$-functions $F_{1}, F_{2}, F_{3}$ on $\mathbb{R}$ with compact supports such that $F_{1}(x)+$ $F_{2}(x)+F_{3}(x)=1$ for $x \in[0,2 a], 0 \leq F_{j} \leq 1, \operatorname{supp} F_{2}=[3 a / 4,5 a / 4], \operatorname{supp} F_{3}=$ $[a, 3 a]$, and $F_{1}(x)=F_{3}(2 a-x)$. Put $k_{j} \stackrel{\text { def }}{=} k F_{j}$. We shall prove below that $\Gamma_{k}^{a} \in \mathbb{S}_{p}$ if and only if all three operators $\Gamma_{k_{1}}^{a}, \Gamma_{k_{2}}^{a}$, and $\Gamma_{k_{3}}^{a}$ belong to $\mathbb{S}_{p}$. Now the above considerations can be applied to $\Gamma_{k_{1}}^{a}$ and $\Gamma_{k_{3}}^{a}$. The operator $\Gamma_{k_{2}}^{a}$ should be investigated separately and we shall show that its investigation can also be reduced to the case of Hankel operators.

Note that Rochberg in [6] in the case $p \geq 1$ has also considered a similar splitting $k=k_{1}+k_{2}+k_{3}$. But in the case $p \geq 1$ he has used the fact that the $\mathbb{S}_{p}$-norm of an integral of operators is less than or equal to the integral of norms. This does not hold for $p<1$. Investigating the operators $\Gamma_{k_{2}}^{a}$, Rochberg has used the fact that the diagonal matrix entries of an operator of class $\mathbb{S}_{p}$ belong to $l^{p}$ which is also wrong for $p<1$.

To prove that the above splitting also works for $p<1$ we need a general result on the so-called Schur multipliers on $\mathbb{S}_{p}$. Recall that each operator $R$ on $L^{2}\left(\mathbb{R}_{+}\right)$ of class $\$_{p}$ with $p \leq 2$ can be represented as an integral operator

$$
(R f)(x)=\left(R_{u} f\right)(x)=\int_{0}^{\infty} u(x, y) f(y) d y,
$$

where $u \in L^{2}\left(\mathbb{R}_{+} \times \mathbb{R}_{+}\right)$is the kernel of $R$.

Now, given a function $\varphi$ on $\mathbb{R}_{+}$we can define the following transform $\mathscr{M}_{\varphi}$ on $\mathbb{S}_{p}\left(L^{2}\left(\mathbb{R}_{+}\right)\right)$:

$$
\mathscr{M}_{\varphi} R_{u}=R_{v}, \quad v(x, y) \stackrel{\text { def }}{=} \varphi(x+y) u(x, y) .
$$


LEMMA 1. Let $\varphi$ be a $C^{\infty}$-function on $\mathbb{R}_{+}$with compact support in $(0, \infty)$. Then $\mathscr{M}_{\varphi}$ is bounded on $\mathbb{S}_{p}$ for any $p \in(0, \infty)$.

We postpone the proof of Lemma 1 and first show how to obtain from it the proof of Theorem 2.

PROOF OF THEOREM 2. We apply Lemma 1 to the transform $\mathscr{M}_{F_{2}}$ and $\mathscr{M}_{F_{3}}$. It is easy to see that

$$
\mathscr{M}_{F_{2}} \Gamma_{k}^{a}=\Gamma_{k_{2}}^{a} \text { and } \mathscr{M}_{F_{3}} \Gamma_{k}^{a}=\Gamma_{k_{3}}^{a} .
$$

It follows from Lemma 1 that $\Gamma_{k}^{a}$ belongs to $S_{p}$ if and only if $\Gamma_{k_{1}}^{a}, \Gamma_{k_{2}}^{a}$, and $\Gamma_{k_{3}}^{a}$ belong to $\mathbb{S}_{p}$. Now supp $k_{3} \subset[a, 2 a], \operatorname{supp} k_{1} \subset[0, a]$, and so by (4) and (5) we have

$$
\begin{aligned}
& \Gamma_{k_{1}}^{a} \in \mathbb{S}_{p} \Leftrightarrow \sum_{j \leq 0} 2^{-|j|}\left\|\mathscr{F}\left(\nu_{j} k_{1}\right)\right\|_{L^{p}}^{p}<\infty, \\
& \Gamma_{k_{3}}^{a} \in \mathbb{S}_{p} \Leftrightarrow \sum_{j \geq 0} 2^{-|j|}\left\|\mathscr{F}\left(\nu_{j} k_{3}\right)\right\|_{L^{p}}^{p}<\infty .
\end{aligned}
$$

Now it remains to investigate $\Gamma_{k_{2}}^{a}$.

LEMMA 2. Let $0<p<\infty$ and $g$ be a function on $\mathbb{R}$ with support in $[3 a / 4,5 a / 4]$. Then $\Gamma_{g}^{a}$ belongs to $\mathbb{S}_{p}$ if and only if $\mathscr{F}_{g}$ belongs to $L^{p}(\mathbb{R})$.

Let us first complete the proof of Theorem 2. It follows from Lemma 2 that $\Gamma_{k}^{a}$ belongs to $\mathbb{S}_{p}$ if and only if $\mathscr{F}\left(F_{2} k\right)$ belongs to $L^{p}(\mathbb{R})$ and the right-hand sides of (6) and (7) are finite. Note that for $j \geq 2$ we have $\nu_{j} k_{3}=\nu_{j} k$ and for $k \leq-2$ we have $\nu_{j} k_{1}=\nu_{j} k$. Now it remains to show that

$$
\sum_{j=-1}^{1}\left\|\mathscr{F}\left(\nu_{j} k\right)\right\|_{L^{p}}^{p}<\infty
$$

if and only if

$$
\begin{gathered}
\left\|\mathscr{F}\left(\nu_{-1} k_{1}\right)\right\|_{L^{p}}^{p}+\left\|\mathscr{F}\left(\nu_{0} k_{1}\right)\right\|_{L^{p}}^{p}+\left\|\mathscr{F}\left(k_{2}\right)\right\|_{L^{p}}^{p} \\
+\left\|\mathscr{F}\left(\nu_{0} k_{3}\right)\right\|_{L^{p}}^{p}+\left\|\mathscr{F}\left(\nu_{1} k_{3}\right)\right\|_{L^{p}}^{p}<\infty .
\end{gathered}
$$

But if we recall that $k_{j}=F_{j} k, j=1,2,3$, we can reduce the above assertion to the following well-known fact (see [3]):

Let $\varphi$ be a $C^{\infty}$-function on $\mathbb{R}_{+}$with compact support, $0<\alpha<\beta<\infty$. Then for any function $g$ with compact support in $[\alpha, \beta]$ we have

$$
\|\mathscr{F}(\varphi g)\|_{L^{p}(\mathbf{R})} \leq c_{\varphi}\|\mathscr{F} g\|_{L^{p}(\mathbf{R})} .
$$

From (10) it follows easily that both (8) and (9) are equivalent to the fact that

$$
\mathscr{F}\left(k \cdot \sum_{j=-1}^{1} \nu_{j}\right) \in L^{p}(\mathbb{R}) .
$$

Now we proceed to the proof of Lemma 1. The proof of Lemma 1 given in the preprint version of this paper was simplified by S. Janson. We need the following inequality:

$$
\|A+B\|_{\mathbf{S}_{p}} \leq\|A\|_{\mathbf{S}_{p}}^{p}+\|B\|_{\mathbf{S}_{p}}^{p}
$$


for any $0<p \leq 1$ and $A, B \in \mathbb{S}_{p}$ (see [7]). In the proof we shall assume $0<p \leq 1$. For $1<p<\infty$ the result follows easily by duality and interpolation arguments.

PROOF OF LEMMA 1 . Let $R \in S_{p}$ and $R=R_{u}$. We have to estimate the operator $R_{v}$ with $v(x, y)=\varphi(x+y) u(x, y)$.

Suppose that $\operatorname{supp} \varphi \subset[0, T / 2]$. Then without loss of generality we can assume that supp $u \subset[0, T / 2] \times[0, T / 2]$ since otherwise we could replace $u$ by $u \chi$, where $\chi$ is the characteristic function of $[0, T / 2] \times[0, T / 2]$. Define a function $\Phi$ on the unit circle $T$ by $\Phi(\exp (2 \pi i x / T))=\varphi(x), 0 \leq x \leq T$. Then

$$
\varphi(x+y)=\sum_{n \in \mathbf{Z}} \hat{\Phi}(n) \exp (2 \pi i n(x+y) / T) .
$$

Therefore

$$
R_{v}=\sum_{n \in \mathbf{Z}} \hat{\Phi}(n) m_{\alpha_{n}} R_{u} m_{\beta_{n}}
$$

where $\alpha_{n}(x)=\exp (2 \pi i n x / T), \beta_{n}(y)=\exp (2 \pi i n y / T), m_{f}$ is multiplication by $f$ on $L^{2}$. Then by (11)

$$
\left\|R_{v}\right\|_{\mathbf{s}_{p}} \leq \sum_{n \in \mathbf{Z}}|\hat{\Phi}(n)|^{p}\left\|R_{u}\right\|_{\mathbf{S}_{p}}^{p}
$$

It remains to notice that $\sum_{n \in \mathbf{Z}}|\hat{\Phi}(n)|^{p}<\infty$ since $\Phi \in C^{\infty}(\mathbb{T})$.

PROOF OF LEMMA 2. We consider three operators with the same kernel $g(x+y)$. The first one we have to investigate is our operator $\Gamma_{g}^{a}$. The second one is the Hankel operator $\Gamma_{g}$ on $L^{2}\left(\mathbb{R}_{+}\right)$, and the third one is the operator on $L^{2}[a / 4, a]$, which we denote by $\widetilde{\Gamma}_{g}^{a}$. (See the figure.)

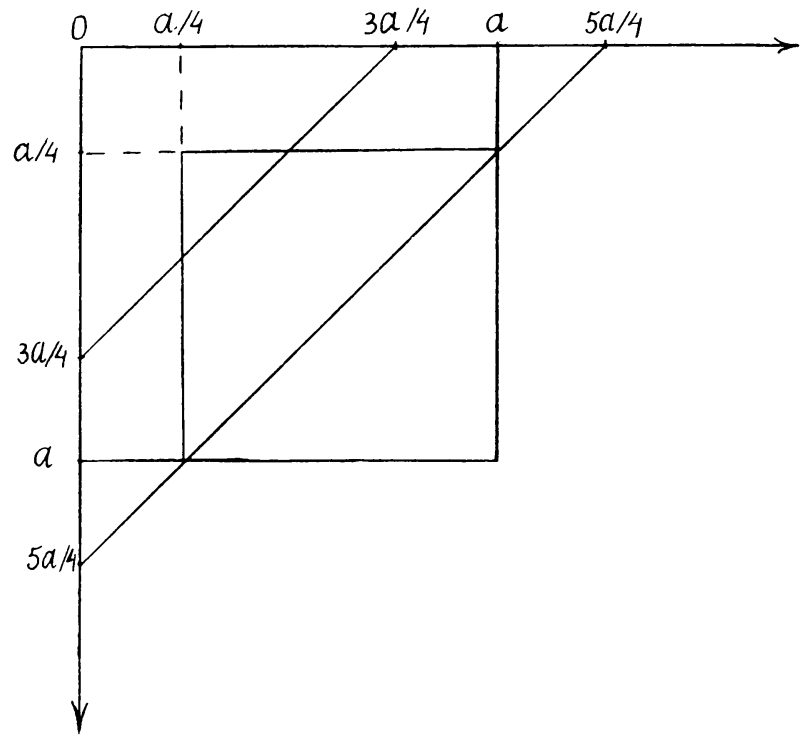

FIGURE 
Let us show first that

$$
\left\|\widetilde{\Gamma}_{g}^{a}\right\|_{\mathrm{s}_{p}} \leq\left\|\Gamma_{g}^{a}\right\|_{\mathrm{S}_{p}} \leq\left\|\Gamma_{g}\right\|_{\mathrm{S}_{p}} .
$$

Indeed, obviously

$$
\Gamma_{g}^{a}=\mathscr{P}_{0}^{a} \Gamma_{g} \mid L^{2}[0, a] \quad \text { and } \quad \widetilde{\Gamma}_{g}^{a}=\mathscr{P}_{a / 4}^{a} \Gamma_{g}^{a} \mid L^{2}[a / 4, a],
$$

where $\mathscr{P}_{\alpha}^{\beta}$ is the orthogonal projection onto $L^{2}[\alpha, \beta]$. The above identities obviously imply (12).

Now $\left\|\Gamma_{g}\right\|_{\mathbf{s}_{p}}$ is equivalent to $\|\mathscr{F} g\|_{L^{p}(\mathbf{R})}$ since supp $g$ is separated away from zero and infinity. Obviously $\left\|\widetilde{\Gamma}_{g}^{a}\right\|_{\mathbf{S}_{p}}=\left\|\Gamma_{\tilde{g}}\right\|_{\mathbf{S}_{p}}$ where $\tilde{g}(x)=g(x+a / 4)$. Again $\left\|\Gamma_{\tilde{g}}\right\|_{\mathbf{S}_{p}}$ is equivalent to $\|\mathscr{F} \tilde{g}\|_{L^{p}(\mathbf{R})}$. This completes the proof.

3. Real interpolation of the spaces $\mathrm{WH}_{p}$. In this section we answer a question by Rochberg [6]. Namely, we show that the spaces $\mathrm{WH}_{p}$ defined in $\S 1$ form an interpolation scale.

THEOREM 3. Let $0<p_{0}<p_{1}<\infty$. Then for $0<\theta<1$

$$
\left(\mathrm{WH}_{p_{0}}, \mathrm{WH}_{p_{1}}\right)_{\theta, p}=\mathrm{WH}_{p}
$$

where $1 / p=(1-\theta) / p_{0}+\theta / p_{1}$.

Let us sketch the proof. We shall use a "retract argument". Consider nonnegative $C^{\infty}$ functions $F_{0}$ and $F_{1}$ on $[-2 \sigma, 2 \sigma]$ such that $F_{0}+F_{1} \equiv 1$ on $[-2 \sigma, 2 \sigma], F_{0} \equiv 1$ on $[-2 \sigma,-\sigma / 2], F_{1} \equiv 1$ on $[\sigma / 2,2 \sigma], \operatorname{supp} F_{0} \subset[-2 \sigma, \sigma / 2]$, $\operatorname{supp} F_{1} \subset[-\sigma / 2,2 \sigma]$.

Now we define an operator $J$ which associates with a kernel $k$ of $W_{\sigma, k}$ a pair of functions as follows. Let

$$
\begin{aligned}
& k_{0}(x)= \begin{cases}\left(F_{1} k\right)(x-2 \sigma), & 0 \leq x \leq 4 \sigma \\
0, & \text { otherwise }\end{cases} \\
& k_{1}(x)= \begin{cases}\left(F_{2} k\right)(2 \sigma-x), & 0 \leq x \leq 4 \sigma, \\
0, & \text { otherwise }\end{cases}
\end{aligned}
$$

Let $f_{0}=\mathscr{F} k_{0}, f_{1}=\mathscr{F} k_{1}$ be the Fourier transforms of $k_{0}$ and $k_{1}$. Then $J k \stackrel{\text { def }}{=}$ $\left\{f_{0}, f_{1}\right\}$. It is easy to see that $k \in \mathrm{WH}_{p}$ if and only if $f_{0}$ and $f_{1}$ belong to $B_{p}$.

Consider now a nonnegative $C^{\infty}$ function $F$ on $\mathbb{R}$ such that $F \equiv 1$ on $[0,5 \sigma / 2]$ and $F \equiv 0$ on $[3 \sigma, \infty)$. Now given a pair of functions $f_{0}$ and $f_{1}$ in $B_{p}$ we define a kernel $k=\mathscr{R}\left\{f_{0}, f_{1}\right\}$ by

$$
k(y)=\left(F \cdot \mathscr{F}^{-1} f_{0}\right)(y+2 \sigma)+\left(F \cdot \mathscr{F}^{-1}\right)(2 \sigma-y), \quad-2 \sigma \leq y \leq 2 \sigma .
$$

It is easy to see that $\mathscr{R}\left\{f_{0}, f_{1}\right\} \in \mathrm{WH}_{p}$ whenever $f_{0}, f_{1} \in B_{p}$. It is also easy to check that $\mathscr{R} J k=k$ for any $k$.

Now the result follows easily from the corresponding fact for Besov spaces:

$$
\left(B_{p_{0}}, B_{p_{1}}\right)_{\theta, p}=B_{p}, \quad 0<\theta<1, \quad 1 / p=(1-\theta) / p_{0}+\theta / p_{1}
$$

(see [3]).

ADDED IN PROOF. A preliminary version of this paper was circulated as Uppsala University, Department of Mathematics Report 1986:9 Recently a Preprint by Pehg Lizhong Hankel operators on the Paley-Wiener space in $\mathbb{R}^{d}$, the Australian National University Research Report CMA-R20-87, has appeared where multi-dimensional analogues of the above results have been obtained. 


\section{REFERENCES}

1. I. Ts. Gohberg and M. G. Krein, Introduction to the theory of linear nonselfadjoint operators in Hilbert space, "Nauka", Moscow, 1965; English transl., Amer. Math. Soc., Providence, R. I., 1969.

2. R. E. A. C. Paley and N. Wiener, Fourier transforms in the complex domain, Amer. Math. Soc. Colloq. Publ., vol. 14, Amer. Math. Soc., Providence, R. I., 1934.

3. J. Peetre, New thoughts on Besov spaces, Duke Univ. Press, Durham, N. C., 1976.

4. V. V. Peller, Hankel operators of class $\mathfrak{S}_{p}$ and their applications (rational approximation, Gaussian processes, the problem of majorizing operators), Mat. Sb. 113 (1980), 538-581; English transl., Math. USSR-Sb. 41 (1982), 443-479.

5. __ A description of Hankel operators of class for $p>0$, an investigation of the rate of rational approximation, and other applications, Mat. Sb. 122 (1983), 481-510; English transl., Math. USSR-Sb. 50 (1985), 465-494.

6. R. Rochberg, Toeplitz and Hankel operators on the Paley-Wiener space, Integral Equations Operator Theory 10 (1987), 187-235.

7. S. Yu. Rotfel'd, Remarks on singular numbers of the sums of completely continuous operators, Funktsional. Anal. i Prilozhen. 1 (1967), 95-96. (Russian)

8. S. Semmes, Trace ideal criteria for Hankel operators and applications to Besov spaces, Integral Equations Operator Theory 7 (1984), 241-281.

LeningRad BRANCh, Steklov Institute of Mathematics, ACAdemy of SCIENCES OF THE USSR, FONTANKA 27, 191011 LENINGRAD, USSR 